\title{
Aristotle on Necessary Principles and on Explaining X Through the Essence of X
}

\author{
Lucas Angioni \\ Department of Philosophy, University of Campinas
}

\begin{abstract}
I discuss what Aristotle means when he say that scientific demonstration must proceed from necessary principles. I argue that, for Aristotle, scientific demonstration should not be reduced to sound deduction with necessary premises. Scientific demonstration ultimately depends on the fully appropriate explanatory factor for a given explanandum. This explanatory factor is what makes the explanandum what it is. Consequently, this factor is also unique. When Aristotle says that demonstration must proceed from necessary principles, he means that each demonstration requires the principle that is the necessary one for the fully appropriate explanation of its explanandum. This picture also provides a key to understand Aristotle's thesis that scientific explanation depends on essences: it is the essence of the attribute to be explained (rather then the essence of the subject-term within the explanandum) that should be stated as the fully appropriate explanatory factor.
\end{abstract}

Keywords: essentialism, explanation, necessity

\section{Introduction}

Recent discussions of the explanatory role of essences in Aristotle's essentialism have focused on the ability of the essence of $X$ to explain (at least some of) the necessary properties of $X{ }^{1}$ One point is crucial in these discussions: what Aristotle means in Posterior Analytics (henceforth APo) when he says that the principle for a scientific demonstration must be necessary. I will argue that what he means is that the principle is the one required for the fully appropriate explanation of a given explanandum. This unorthodox reading allows that, in some cases, the essence of a subject will not on its

Corresponding author's address: Lucas Angioni, University of Campinas, Department of Philosophy, Rua Cora Coralina s/n, 13083-896, Campinas-SP, Brazil. Email: angioni.lucas@gmail.com.

(Lloyd 1981), (Fine 1994), (Charles 2000, 2010), (Peramatzis 2010, 2011), (Williams and Charles 2013). 
own deliver the fully appropriate explanation for some of its features, even if it contributes to that explanation. Instead, the fully appropriate explanation will be delivered either by the essence of the feature itself or by the essence of the explanandum as such. This account of the notion of a necessary principle has several philosophical advantages: it pays attention to the different ways in which Aristotle talks about necessity, allows us to attain a consistent overall picture of Aristotle's project in APo, and avoids charging him with blatantly false views when discussing the role of necessity in scientific explanations.

\section{Ways of Talking about Necessity and Necessary Items}

\subsection{Necessity-1}

One of the ways in which Aristotle talks about necessity in APo involves the necessary character of the consequence captured in a demonstration. This notion of necessity clearly relates to the mere logical passage from premises to conclusion, which, far from being specific to scientific demonstration, is a general trait of each valid deduction. It is an important question whether Aristotle's notion of syllogistic consequence can be fully analysed as a mere logical consequence or relies on some extra-logical requirements, but I need not discuss this question in any detail ${ }^{2}$ because it is clear that it is not included among the characteristic features in virtue of which a syllogism is turned into a scientific demonstration (see APo 71b23-24, 74b15-18).

\subsection{Necessity-2}

Another way in which Aristotle talks about necessity or necessary items in $A P o$ is related to the necessary character of the sentences that constitute a demonstration. Scholars have argued that the notion of a necessity sentence should be understood as a specific sort of predicative tie, instead of being generated by some kind of propositional modal operator applied to a previous sentence. ${ }^{3}$ This notion of a necessary sentence plays a role in Aristotle's project in $A P o$, but it is not the most important feature of it. It is probably correct to say that a scientific demonstration must be constituted of sentences which are necessarily true. ${ }^{4}$ Yet, being constituted of necessary sentences is not sufficient for turning a sound syllogism into a demonstration. Nonetheless, Aristotle seems to be saying so at APo 74b15-18:

${ }^{2}$ For discussion see (Corkum forthcoming).

3 (Malink 2013, 27).

4 I say "probably" as a way of pointing to the problem of sentences that are true not necessarily but for the most part (see I 30, 87b21-25; II.12, 96a8-19). See section (3.5) about this. 
(T1) Therefore, the syllogism must proceed from necessary [items], from true [items] you can deduce without demonstrating, but from necessary [items] you cannot deduce without demonstrating-this is precisely the mark of demonstration. (74b15-18, Barnes's translation slightly modified).

As Barnes $(1993,126)$ has pointed out, Aristotle seems to be committed to the false proposition that "if $P$ is inferred from $\Pi$, and $\Pi$ is necessary, then $P$ is demonstrated". ${ }^{5}$ Now, my interpretation does not commit Aristotle to this false proposition. Let me call it henceforth BFP (Barnes's False Proposition). And the key to understanding how Aristotle is not committed to BFP is to realize that his talk about the "necessity of the principles" in APo does not focus on the notion of a necessary predicative sentence.

\subsection{Necessity-3}

Aristotle in APo also talks about the necessary character of the principles from which a demonstration must proceed $\left(74 b_{5}-6\right)$. Most scholars seem to conflate this third way with the second one. ${ }^{6}$ They think the necessary character of a principle collapses into its necessary truth as a sentence. If principles are interpreted as predicative sentences, their necessity qua principles amounts (according to those scholars) to the necessity of the predicative relation between its subject and its predicate. But, in my view, this conflation is wrong and, besides other things, it cannot avoid attributing $B F P$ to Aristotle. My claim is that, even if it is indeed true that the predicative relation between the subject and the predicate is a necessary one when a given predicative sentence is a principle for a demonstration, this is not the point Aristotle is making when he urges that demonstrations must proceed from necessary principles (74b15-18 $=\mathrm{T} 1,71 \mathrm{~b}_{12}, 73 \mathrm{a} 24,74 \mathrm{~b} 5-6,75 \mathrm{a} 12-15,88 \mathrm{~b}_{31-32}$ ).

The third way in which Aristotle talks about necessary items in APo introduces the notion of a principle that is the necessary one for the fully appropriate explanation of a given explanandum. This notion of a necessary principle is compatible with the necessary character of the sentences that constitute a demonstration. What is labelled a necessary principle might itself be a necessary predicative sentence. However, the necessity at stake, when such a sentence is labelled a necessary principle, cannot be reduced to (and understood in terms of) the necessary truth of its predicative tie. The necessity attributed to a demonstrative principle as a principle rests on the notions of explanatory relevance and full explanatory appropriateness. Be-

5 This proposition is false because it states that Necessity-2 is sufficient for demonstrations. See (Mignucci 2007, 171), (Hankinson 1998, 161).

6 (Barnes 1993, 125-127), (Ross 1949, 528), (McKirahan 1992, 125-126), (Mignucci 2007, 171), (Lloyd 1981, 158), (Burnyeat 1981, 110). 
ing a necessary principle amounts to being the principle that is the necessary one for attaining the fully appropriate explanation of a given explanandum. This way of understanding the notion of a necessary principle avoids charging Aristotle with $B F P$ : "if $P$ is inferred from $\Pi$, and $\Pi$ is necessary, then $P$ is demonstrated". What Aristotle is saying at $74 \mathrm{~b}_{15}-18$ is rather that "if $P$ is inferred from $\Pi$, and $\Pi$ is what is strictly necessary for the fully appropriate explanation, then $P$ is demonstrated".

Before discussing some of the key passages in support of the view that Aristotle is committed to such a notion of Necessity-3, I should stress-to forestall confusion about terminology-that the notion of a fully appropriate explanation of a given explanandum is exactly Aristotle's notion of scientific demonstration. Aristotle's demonstrations cannot be reduced to sound deductions concerned with justifying and certifying our beliefs about the truth of a given proposition. ${ }^{7}$ Aristotle's demonstrations are not even primarily concerned with establishing in the first place that some problematic sentences are true. I add that a strictly scientific demonstration-which expresses scientific knowledge simpliciter (71b9-12, cf. 74a32-33, 76a13-15) cannot be satisfied with some reasonable explanation. The essential feature of a demonstration is to display the most relevant explanatory factor of a given explanandum that is already assumed to be the case. ${ }^{8}$ And the most relevant explanatory factor-which is indeed unique-is a cause (in the Aristotelian sense of "cause") that makes the explanandum what it is. Let it be clear, then, that I use here the words "demonstration" and "explanation" according to the picture outlined in this and the next section.

\section{The Notion of a Necessary Principle}

\subsection{Bearers of the Adjective "Necessary"}

It is important to stress that, when Necessity- 3 is involved, the bearers of the adjective "necessary" (or equivalent expressions, like "cannot be otherwise", 71a12) in Aristotle's Greek are items such as "principles" (74b5-6), "middle term" (75a13), "premise" (89a4) and-more controversially-causal relations (71b10-12). There are explicit occurrences of expressions such as "necessary principles" (74b5-6) and "necessary middle term" (75a13), but I hold that also many absolute occurrences of the adjective "necessary" (with no substantive uncontroversially implied in the context) must be taken in the same way in APo I.6. More precisely, the expression "ex anankaion" at 74b15, 17, 18 and 26 (as well as at 73a24) points to the same claim made at the beginning

7 See (Taylor 1990, 116-117), (Kosman 1973), (Burnyeat 1981, 108-115), (McKirahan 1992, 26 32), (Matthen 1981, 4-10), (Fine 2010), (Lesher 2001, 46), (Goldin 2013).

8 A similar conception can be found in (Koslicki 2012, 189, 194-201). 
of APo I.6, namely, that a scientific demonstration must proceed from principles that are necessary qua principles for the intended explanation. Remember that at $74 \mathrm{~b} 15$ we find the first sentence of $\mathrm{T} 1$, which, if interpreted in terms of Necessity-2, generates BFP. I have suggested that $\mathrm{T} 1$ should be interpreted in terms of Necessity- 3 in order to avoid BFP. Thus, Aristotle's use of the expression "ex anankaion" is consistent in all those passages in APo I.6 (some of them will be discussed below). His use of this expression does not introduce the requirement that a demonstration must be constituted of necessary predicative sentences (even if Aristotle does accept this requirement). It rather introduces a requirement about the appropriateness of an explanatory principle as a principle. I will discuss the key passages in section 3.5, but first I will clarify the notion of Necessity-3 further, and explain why this notion does not commit Aristotle to any blatantly false proposition like BFP.

\subsection{The Use of "Necessary" as Adjective of "Principle"}

When the adjective "necessary" in Aristotle's Greek is connected with the term "principles" ( $\left.74 \mathrm{~b}_{5}-6\right)$, it takes the principle at stake as a principle and indicates a specific way in which the principle performs its function as a principle in a given explanatory context. After all, the bearer of the complex predicate "necessary principle" is a sentence $S$. But sentence $S$ is not called "necessary", and then, a "principle", as if these two predicates were independent of each other and just happened to be lumped together. The application of the predicate "necessary" to a sentence $S$, in this case, presupposes the prior application of the predicate "principle" to the same sentence. A sentence $S$ is called a principle with regard to the explanatory work it performs in a given explanatory context; the second predicate "necessary" is, then, applied to the same sentence $S$, but taking $S$ as a principle and indicating the specific way in which it is a principle.

A comparison with a case explicitly discussed by Aristotle might be in order here. As the adjective "good" in the composite predicate "good cobbler" presupposes that its subject is a cobbler and indicates a specific way in which she exercises her ability as a cobbler," ${ }^{9}$ so also the adjective "necessary" in the expression "necessary principle" presupposes that its subject is a principle and indicates a specific way in which it performs its role as a principle. Therefore, as the correct paraphrase of "good cobbler" when ap-

9 On Interpretation, 2ob31-36. It is hard to understand Aristotle's point (see Ackrill 1963, 146-148), but what is important to me is his acknowledgement at 2ob35-36 that "good cobbler" is not used as an equivalent to a sum of predicates in which both "good" and "cobbler" would preserve the same meaning they have when applied separately to a given subject. 
plied to Victoria will be something like "Victoria is good at mending shoes etc" (rather than "Victoria is able to mend shoes and is a very good character of a person"), so also the correct paraphrase of "necessary principle" when applied to a given sentence $S$ will be something like " $S$ is the principle necessary for the fully appropriate explanation of that explanandum" (rather than " $S$ has some explanatory power in a given context and is a necessarily true sentence"). Note that it might be true that Victoria is a person of good character, and that the sentence $S$ is necessarily true. However, these truths are not part of the meaning of the expressions at stake. Even if Victoria is a good person, when someone says that she is a good cobbler, he is saying that Victoria is good (i.e., skillfull, efficient) at mending shoes. In the same way, even if a sentence $S$ is necessarily true, when a scientist declares that $S$ is the necessary principle for a demonstration, she is saying that the sentence $S$ is the principle necessary for the fully appropriate explanation of a given explanandum. ${ }^{10}$

The same applies to "necessary middle term". The meaning of this expression at 75a13 is not "a middle term that is necessarily attributed to its subject", even if it happens to be necessarily attributed to its subject. The expression "necessary middle term" takes the middle term as a middle term or, more precisely, as a demonstrative middle term (i.e. as introducing an explanatory claim in an intended demonstration) and indicates the way in which this middle term performs its job as an explanatory middle term. The expression means that the middle term is the necessary one for the fully appropriate explanation of its explanandum. ${ }^{11}$

\subsection{The Triadic Structure Involved in a Necessary Principle}

The explanatory context involved in the use of "necessary principle" has a threefold structure. The explanandum as such (even if one can refer to it in the ordinary language by means of a nominal expression) must be formulated as a predicative relation between a subject and an attribute. What one wishes to explain is, for instance, why a certain kind of noise occurs in the

${ }^{10}$ Other cases in which the adjective "necessary" has nothing to do with being necessarily true are found in Topics (155b19, 20, 29, 36; 156a10; 157a12; 159a2o; maybe 162b1; 161b29) and Prior Analytics (42a39; 47a19; 53a35; 66a36). To call a given sentence $S$ "a necessary premise" (as at 155b19) amounts to saying that $S$ is the premise required (in a given argumentative context) for the intended valid conclusion.

${ }^{11}$ A similar story holds for $74 \mathrm{~b} 29-30$ : Aristotle is saying that "the middle term $B$ is not necessarily [sc. the middle term for the appropriate demonstration]; [...] for it is possible [sc. for that middle term actually used in the attempted demonstration] not to be [sc. the middle term in the desired explanation, namely, the one that captures the why]". I will skip the convoluted paragraph 75a1-11, but I submit that all occurrences of "necessary middle term" (75a2, 4, 8-9) can still be understood in the same way as the occurrence at 75 a13. 
clouds, or why being long-lived is attributed to quadrupeds, or why being mortal is attributed to humans. There is an ordinary name for the occurrence of this kind of noise in the clouds, "thunder", while there is no ordinary name for the occurrence of the attribute long-lived in quadrupeds. But this latter fact does not affect the possibility of introducing the explanandum through a nominal expression like "quadruped's longevity". The important point is that behind the nominal expression there is a complex structure which should be articulated by means of a predicative tie. Every explanandum should be cast in the predicative formula "why does $A$ belong to $C$ ". ${ }^{12}$

In reference to this predicative explanandum, the necessary-3 principle is understood as a middle term $B$ in the syllogistic framework ${ }^{13}$ of explanatory demonstrations. This middle term introduces a more basic attribute that, belonging to the same subject $C$, makes $C$ have the attribute to be explained, $A$. With regard to these features I hold that the notion of a necessary principle is triadic, not dyadic. ${ }^{14}$ The relevant contrast is with the notion of a necessary predicative sentence which is merely dyadic: a given sentence $S$ 's being a necessary-2 sentence is a matter of how its predicate is related to its subject, so that one can ascertain whether or not $S$ is necessary-2 by focusing only on that predicative tie. There is no need to consider the relation of $S$ to other sentences. But a middle term's being a necessary-3 principle is a matter of how the middle term is related to a given explanandum which is itself formulated as a predication. The middle term $B$ is a predicate that belongs to $C$, but what makes it a demonstrative middle term is its explaining why $C$ has the attribute $A$, and this explanatory work depends not only on the predicative tie between $B$ and its subject $C$, but also on the predicative tie and the explanatory relation between $B$ and $A$. Consequently, in order

${ }^{12}$ See (Williams and Charles 2013, 123), (Charles 2010, 289-296), (Charles 2000, 213-220). From now on, I will follow standard use of " $A$ " for the attribute to be explained, " $C$ " for the subject of which that attribute is predicated, and " $B$ " for the middle term explaining why $A$ belongs to $C$.

${ }^{13}$ I will not discuss whether the syllogistic framework is suited to Aristotle's notion of demonstration. The standard anti-syllogism position is found in (Barnes 1981). For discussion, see (McKirahan 1992, 25, 149-163), (Smith 1984), (Ferejohn 1994, 83-84). My view is that (i) demonstrations are concerned with capturing the appropriate explanatory factor for an explanandum expressed in predicative form, (ii) such explanations can be cast in the triadic framework of syllogisms, (iii) so the syllogistic framework is thereby suited to demonstrations-even if syllogisms are not suited as a tool for scientific research.

${ }^{14}$ I use "dyadic" here, in contrast with "triadic", in attention to the fact that an Aristotelian predicative sentence is a dyadic tie between a predicate and a subject (see APr 24a16-17). My usage has nothing to do with predicates like "being the father of", which is a "dyadic predicate" in the sense that it must be applied to two subjects in order to get a complete sentence. The same holds of my usage of "triadic": I am highlighting the fact that an explanation has a threefold structure, so that an explanatory factor cannot be designated without taking into account the triplet of terms involved in that structure. 
to ascertain that a given middle term $B$ is a necessary-3 principle, one cannot restrict her focus on the predicative tie of $B$ and its subject $C$ with no attention to the attribute $A$ which the middle term as a principle is meant to explain. One must rather focus on the triadic relation between the middle term $B$ and the predicative explanandum, $C A .^{15}$

\subsection{Explanandum-sensitiveness}

The notion of a necessary principle is explanandum-sensitive. For a given explanandum $C A$, a given middle term might be the necessary principlethe principle required for the fully appropriate explanation of that explanandum. However, nothing guarantees that the same middle term will preserve its character of necessary principle for a different explanandum $C A^{\star}$ (the attribute $A^{\star}$ being different from $A$ ).

This point is highly important for a more fine-grained understanding of the explanatory role of essential predicates. In most versions of Aristotle's essentialism, essential predicates are charged with the responsibility of explaining on their own most of the necessary predicates of their subjects. ${ }^{16} \mathrm{I}$ will argue that their essentiality might still be insufficient for the fully appropriate explanation of why their subject has or must have some of its necessary features.

A full analysis of what being a necessary principle amounts to is beyond the scope of this paper. ${ }^{17}$ For my purposes, it is enough to characterize the notion of a necessary principle - the principle delivering the fully appropriate explanation of its explanandum - with three features: (a) being able to guarantee (in the appropriate context) a sound deduction of its explanandum; ${ }^{18}$ (b) being also a sine qua non condition for its explanandum to obtain;

${ }^{15}$ I will write $C A$ (instead of $A C$ ) to depict the pragma consisting in $A$ being attributed to $C$. Nothing important hinges on the order between $C$ and $A$, I just prefer $C A$ because it is nearer to formulae in ordinary language.

${ }^{16}$ In some versions of essentialism, essential predicates are discharged of that responsibility: the explanation of the necessary predicates of a subject is not performed by the subject's essence on its own, but also requires the essences of others objects involved in the explanatory story. See (Peramatzis n.d.) and (Koslicki 2012, 202-206). However, these versions do not account for the case which seems to be central in Book II of APo, namely, the case in which A's essence or CA's essence (for instance, thunder's essence) is the necessary principle.

${ }^{17}$ Full analysis of this subject will require close examination of Aristotle's criteria for explanatory appropriateness in APo I.4-5, I.7, I.9 and I.13, with discussion of other interrelated points like coextensive katholou predicates, the notion of suggeneia ("appropriate relatedness"), the relevance operator "as such", the primitiveness of the primary cause, etc.

${ }^{18}$ The appropriate context is already encapsulated in the fact that the term at stake is taken as a demonstrative middle term, which implies that (i) it has a predicative connection with $C$, (ii) it has a predicative connection with $A$, (iii) it has some claim for being the cause of 
(c) being the exactly appropriate explanatory factor that makes its explanandum be what it is. In order to be a necessary principle, an explanatory factor (expressed as a middle term in a syllogism) must display all these three features. ${ }^{19}$ Note that the feature (c) is such that its fulfilment entails the fulfilment of both other features, i.e. (a) and (b). If a middle term $B$ is the appropriate explanatory factor that makes its explanandum what it is, it is thereby a sine qua non condition for its explanandum to obtain and guarantees a sound deduction of it. The real advantage of unpacking the notion of a necessary-3 principle into these three features is that it helps us understand what is wrong when a seemingly good explanation is not the most appropriate one.

A few foil examples are in order. I will first consider a case in which the three requirements are fulfilled, and then contrast it with the case in which two requirements are not met. Take $C$ as "human" and $B$ as "rational animal". Take $A$ as "capable of becoming literate". On Aristotle's view, being a rational animal is an essential feature that appropriately explains human's capability of becoming literate. Now, consider another explanandum with a very different attribute: let $A$ be "mortal". If we grant that the most appropriate explanation must pick up the explanatory factor that precisely makes its explanandum what it is, and if we grant that mortality depends, on Aristotle's view, precisely (and ultimately) on facts about the elemental matter that constitute sublunary living beings (cf. De Caelo 288b15-18), we can see that being a rational animal is not any more the most appropriate explanation for human mortality. Human mortality has some important connection with human essence as rational animals. However, being a rational animal is not the most relevant explanatory factor that makes mortality be what it is (i.e., mortality does not depend primarily on human rationality for being what it is) and, besides, being a rational animal is not even a sine qua non condition for being mortal: humans could still be mortal even if they were not rational animals. ${ }^{20}$ Thus, requirements (b) and (c) described above are

$C$ 's being $A$. These remarks avoid some problems addressed by Koslicki $(2012,194)$, when she argues that the inference from her (2.a) to her (2.b) will not be logically valid without supplying an auxiliary premise.

${ }^{19}$ Features (a) and (b) taken together amount to the requirement that a principle must be a necessary and sufficient condition for its explanandum to obtain. Put in syllogistic framework, this requirement is equivalent to the requirement that the middle term must be coextensive with the major term. Now, Aristotle argues very carefully that conditions (a) and (b) together are not enough for an appropriate explanans (APo I.13). See (Koslicki 2012, 198-199), (McKirahan 1992, 214-216), (Charles 2010, 308). This point only stresses the importance of feature (c), which is not reducible to any extensional feature of the relations between the three terms.

${ }^{20}$ On Aristotle's view, humans will not be humans if they were not rational animals. However, this would not prevent Aristotle from engaging in counterfactuals designed to highlight 
not satisfied in this case.

Before exploring some further points, consider another foil example, Aristotle's case of two lines perpendicular to a third line (APo 74a13-16). Take $C$ as "two lines cutting a third line", $A$ as "do not meet" and $B$ as "producing right angles as they cut the third line". Now, B's being attributed to $C$ is enough for soundly deducing that $A$ is attributed to $C ; ;^{21}$ after all, given that those lines produce right angles as they cut the third line, they do not meet. Nonetheless, producing right angles is not the appropriate feature to explain why those lines do not meet each other (cf. Barnes 1993, 123). It is not important for the explanation that the angles produced are right ones; if those lines produced acute or obtuse angles, it would remain true that they do not meet each other, provided that the two angles are equal to each other in any way. Aristotle's point is that producing right angles does not deliver the most appropriate explanation: the fully appropriate explanation requires the angles to be equal to each other in whatever way, and that is all. And "being equal to each other in whatever way" does not include any mention of specific ways in which the two given angles are equal to each other, e.g. by being right (or acute or obtuse) angles. The explanandum at stake does not primarily depend on the angles being right (nor does it depend on the angles being acute or obtuse). The necessary principle, in this case, will be "producing angles (as they cut the third line) equal to each other in whatever way". 22

Another example: take $C$ as "isosceles triangle", $A$ as " $2 R$ " (i.e., "having the sum of its internal angles equal to two right angles") and $B$ as "being a rectilinear plane figure enclosed by three lines with two sides (or angles) equal to each other". Now, the Barbara-NNN relating these three terms would, of course, deliver a sound deduction of the predicative sentence taken as explanandum, but the part of the $B$-term which I have put in italics smuggles an irrelevant factor into the explanation. That isosceles triangles are isosceles, i.e., have two sides (and two angles) equal to each other, is not relevant for them in order to have $2 \mathrm{R}$. The fully appropriate explanation of the predicate $2 \mathrm{R}$ applied to its proper subject requires only the features that make the subject a triangle, $s c$. a rectilinear plane figure enclosed by three lines.

that rational animal is not even a sine qua non condition for mortality to obtain. Aristotle uses such counterfactuals when he discusses some features of primary causes in APo I.13, 78b16-17.

${ }^{21}$ What I mean by "being enough for soundly deducing" is "being enough for soundly deducing in the appropriate circumstance, namely, with the aid of the major premise which disentangles the entailing relation from $B$ to $A$ ". Now, this appropriate circumstance is already encapsulated in the fact that $B$ is taken as the middle term.

${ }^{22}$ This is clearly what Aristotle intends with "in so far as they are equal in any way at all" at 74a16 (even if some details of the proof he has in mind are obscure). See (Barnes 1993, 123). 
Consequently, in this case, being a rectilinear plane figure enclosed by three lines will be the necessary principle for explaining why isosceles triangles have $2 \mathrm{R}$ (cf. APo $85 \mathrm{~b} 4-13$ ). ${ }^{23}$

Now, it is important to remark that, in the three foil examples above, the middle terms "rational animal", "producing right angles as they cut the third line" and "being a rectilinear plane figure enclosed by three lines with two sides (or angles) equal to each other" are similar as middle terms for the intended explanations. All those terms, as middle terms (i) are essential predicates of their respective subjects and introduce their whole essence; (ii) deliver a sound deduction of the predication assumed as explanandum; (iii) but, in advancing the whole essence of their subjects as explanatory factors, fail to present the most appropriate explanation of their respective explananda.

What is wrong with the explanations delivered by those middle terms, which are essential predicates of their respective subjects? Among requirements (a)-(c) for being a necessary-3 principle, those middle terms satisfy only (a): they ensure a sound deduction of their explananda. But they do not even count as a sine qua non condition for their explananda to obtain, i.e. requirement (b) is not met, let alone as the fully appropriate explanatory factor for them, i.e. requirement (c) is not fulfilled either. They still deliver some good explanation for their explananda, but this is not enough on Aristotle's view: in order to count them as necessary-3 principles, Aristotle wants them to deliver the most appropriate explanation.

Requirement (c) is basic: a middle term must be the exactly appropriate explanatory factor that makes its explanandum $C A$ (not its subject $C$ ) what it is. Aristotle is focusing on what the explanandum exactly is rather than on the sole essence of the subject $C$. This is Aristotle's meaning when he applies the Greek expressions normally translated as "in itself" (or "as such") to the "object" of scientific knowledge. Now, "object" might be misleading here: it might lead us to believe that Aristotle's focus is on the subject $C$. However, it is rather the pragma $C A$ that counts as "what one is concerned with scientifically knowing" ${ }^{24}$ And what is a pragma in itself or as such? Even if

${ }^{23}$ Things are a little more complicated than that, because Aristotle insists that isosceles triangle is not the most appropriate subject for $2 \mathrm{R}$ (in APo I 4-5, for instance, in 74a2-3, 16-17, 32-b1). For my purposes, though, it is enough to consider Aristotle's discussion in APo I.24: if your demonstration has started with isosceles as its subject, then it is preferable to avoid selecting its specific description ("as isosceles") as the explanatory factor (85 b5-7). My paraphrase of $85 \mathrm{~b} 5-7$ is the following: "since $2 \mathrm{R}$ belongs [sc. to isosceles] not as isosceles but as triangle, the knower who knows because it is isosceles has less knowledge [of the explanandum as such-of what the explanandum exactly is in itself] than the knower who knows because it is triangle"- "hoti" at 85b6, 7 is "because", not "that", pace Barnes.

${ }^{24}$ The term "pragma" is used in the sense of explanandum with predicative structure in APo 
both $C$ and $A$ are important factors in settling this question (for they are the elements of the pragma $C A$ ), the attribute $A$ plays the most important role in determining what the explanandum is qua the explanandum at stake. If I wish to explain "human mortality", I am thereby concerned with explaining facts about humans in general, but I am more precisely concerned with one specific fact among those, namely, the one about mortality. Thus, the application of Greek expressions equivalent to "as such" (or "in itself") to explananda has the effect of highlighting (or giving expression to) the requirement (c). If I aim at scientifically explaining why $C$ is $A$, I should focus on what $A$ is exactly as such. My middle term $B$ must (first of all) encapsulate what $A$ is in itself, and the encapsulating feature might not be $C$ 's essence. When Aristotle says that "necessary [items] are what belong to an object (pragma) in itself" (74b6-7), he means that "necessary [principles] are what belong to explananda in themselves". Thus, to return to my foil examples, when I pick up animal rationality as human essential feature to explain why humans are mortal, I miss the most relevant factor that makes mortality (for humans as well as for other objects) what it is. I have thereby some explanation of my explanandum, but not the most appropriate one and, therefore, not the scientific one. Similarly, when I pick up isosceles triangle's definition to explain why isosceles triangle has $2 \mathrm{R}$, I do not express thereby any understanding that this attribute does not depend on any specific feature of the sides and angles of a triangle, but only on any triangle's being a three-sided rectilinear figure.

\subsection{Aristotle's Commitment to Necessity-3}

The best evidence to show that Aristotle is committed to the notion of Necessity- 3 is $T 1$, which is quoted in section 2.2. If we invoke Necessity-2 in our interpretation of $\mathrm{T} 1$, we cannot avoid charging Aristotle with BFP: "If $P$ is inferred from $\Pi$, and $\Pi$ is necessary, then $P$ is demonstrated" (Barnes 1993, 126). Since this proposition, besides being clearly false, is not consistent with many other things Aristotle says about demonstration in APo, a different interpretation for $\mathrm{T} 1$ seems to be needed. I am providing such an interpretation with the notion of Necessity- $3 .{ }^{25}$

71b11, 91b14, 93a22, 98b30 (more controversially: 73b28, 74b7; APr 46a25). Cf. (Burnyeat 2011, 19, footnote 57).

${ }^{25} \mathrm{BFP}$ will be incompatible with (i) Aristotle's insistence on the six requirements introduced at 71b20-32, among which the causality requirement occupies the first rank, as well as his insistence that (ii) premises in a demonstration must express per se predicative ties (73b1618, 75a29-31), (iii) principles should be suggenes with (appropriately related to) their explananda (75 $\left.b_{3}-12,76 a 8-9,29-30\right)$, (iv) scientific explanation should state the principles of the explanandum as such $\left(75 \mathrm{~b}_{3} 6-40,76 \mathrm{a} 4-7\right)$, and (v) scientific explanation requires appropriate principles (71b22-23, 72a5-6, 74b25-26). 
However, one might argue that Aristotle consistently employs the notion of necessity in APo along the lines of Necessity-2. The key chapter, APo I.6, seems to insist that demonstrative premises should be necessarily true. Aristotle's remarks at the opening of APo I.4 also seem to invoke the notion of necessarily true premises. Besides, Aristotle's definition of scientific knowledge in APo I. 2 seems to attribute necessity to the proposition that one comes to know scientifically. I will now address these exegetical difficulties.

First of all, I should emphasize that I am not banishing necessary sentences from Aristotle's theory of demonstration. The triadic notion of a necessary-3 principle is compatible with the standard view that a demonstrative sentence is necessarily true, i.e. true by Necessity-2. Only middle terms as middle terms can qualify as necessary principles in the triadic sense; but when cast in the predicative sentences in which they are related to the extremes, the middle terms can still deliver sentences which are themselves necessarily true. This is exactly what happens with "rational animal" as a middle term attributed to humans in order to explain their capability of becoming literate (one of my key examples in the previous sections). Thus, an advantage of my account is that it makes sense of otherwise vexing things Aristotle says about "necessary principles" (in T1 and elsewhere) without abandoning the importance of Necessity-2 for demonstrative knowledge.

A good way of exploring this point is to discuss the occurrence of "necessity" terminology in the beginning of APo I.4. In the central passages of this chapter, Aristotle does indeed deal with the standard notion of a necessary-2 predicative sentence; he is concerned with showing that (at least some kinds of) per se attributes are necessarily attributed to their subjects (73b16-18). But from this it does not follow that Aristotle is talking about the same notion of Necessity-2 in the first paragraph of APo I.4, when he points out that since the object of demonstrative knowledge is necessary, "demonstration is, then, a syllogism from necessary [items]" (73a24). The adjective translated as "necessary" at $73 a 24$ has no noun attached to it, and one might be tempted to assume that Aristotle is talking about predicative sentences as mere predicative sentences ${ }^{26}$-as if he focused on the mere predicative tie between the subject and the predicate with no concern for the explanatory role those sentences perform in a triadic context. Yet, such an assumption goes beyond the direct evidence from the text. I admit that one cannot settle whether Necessity-2 or Necessity-3 is evoked in the key sentence at $73 a 24$ from a purely grammatical standpoint. The decision between one or another kind of necessity should be grounded on the philosophical interpretation of the surrounding context. Now, since commitment to Necessity-2 leads to $B F P$ and since commitment to Necessity- 3 will furnish a good argument that

\footnotetext{
${ }^{26}$ See (Barnes 1993, 111), (McKirahan 1992, 80-83).
} 
also squares with the context of $73 a 24$, I submit that the "necessary [items]" at $73 \mathrm{a} 24$ are necessary-3 principles. Aristotle's point, then, is the following: he stresses that demonstration requires necessary-3 principles ("demonstration is, then, a syllogism from necessary [items]", 73a24), and then goes on to discuss which sorts of predicates are involved in a necessary- 3 principle ("we must settle, then, from what items, i.e., from what kind of items [sc. from what kind of predicate] demonstrations proceed", 73a24-25). Being necessarily-2 true of its subject is a sine qua non, but not a sufficient condition for being a necessary-3 principle; actually, several further requirements which go beyond Necessity-2 are made in APo I.4-5.

Now, one might argue that Aristotle is appealing to the notion of Necessity-2 in his definition of scientific knowledge, but this is controversial. The key passage is this:

(T2) We think we have scientific knowledge of something simpliciter [...] when we think we know of the cause because of which the explanandum holds that it is its cause, and also that it is not possible for it to be otherwise" (71b9-12, Barnes's translation modified).

Scholars have assumed that the pronoun "it" (touto) refers back to "pragma" (explanandum, 71b11) and that pragma should be represented as a mere predicative sentence. However, we can get a much better sense if we understand "touto" as referring back to the phrase "hoti ekeinou aitia esti" ("that [the cause] is the cause of it"). What cannot be otherwise-and what we understand that cannot be otherwise if we have scientific knowledge-is that the cause [sc. the one picked up in our demonstration] is indeed the [sc. most appropriate] cause of the pragma. ${ }^{27}$ Thus, Aristotle can be seen as defining scientific knowledge precisely by this strong requirement about causes (in the Aristotelian sense of "cause"): even if there are many causes that somehow explain our explanandum, there is only one cause that explains it in the most appropriate way, and we only attain scientific knowledge if we attain the most appropriate cause for our explanandum. ${ }^{28}$ Such an interpretation of Aristotle's definition of scientific knowledge agrees perfectly with the notion of a necessary-3 principle as described in my previous sections.

As for Aristotle's talk of necessity in APo I.6, it is far from clear that Aristotle is talking about Necessity-2 throughout the chapter. I have already argued that $\mathrm{T}_{1}$ is the best evidence against restricting ourselves to Necessity-2

27 "Aition" (with no adjective) means primary or most appropriate cause in several passages: $78 \mathrm{a} 26,78 \mathrm{~b} 15,17,98 \mathrm{~b} 17$ (and many other occurrences in APo II. 16).

${ }^{28}$ This notion of scientific knowledge is too strict, too demanding etc., but Aristotle was perfectly aware of it and the difficulties in attaining its requirements did not deter Aristotle from holding it. See 76a26-30. 
in interpreting Aristotle's theory of demonstration. A full discussion of this vexing chapter is beyond the scope of this paper, but I will now argue that a significant number of key passages make better sense on my interpretation. Again, a decision about whether Necessity-2 or Necessity- 3 is involved in most of those passages cannot be settled on purely grammatical criteria. I argue that Necessity-3 delivers a better understanding of Aristotle's philosophical point in those contexts. Let me consider the passage which immediately follows $\mathrm{T}$ :

(T3) There is evidence that demonstrations proceed from necessary [items] in the way in which we bring objections against those who think they are demonstrating: we say that it is not necessary, if we think $[\ldots]$ that it is possible to be otherwise." (74b18-21, Barnes's translation modified)

It is precipitate to assume that the objection "it is not necessary" should be understood as equivalent to "this is not a necessarily true sentence". Aristotle's words can be understood along the lines of Necessity-3. If someone claims that "our economy is good because of the commodity prices", it is open to anyone to object "not necessarily", meaning that "it is not primarily because of the commodity prices that our economy is good", since there are better explanations for that explanandum ("why is our economy good?"); in short, it is possible to explain the explanandum otherwise and in a better manner. An objection of this sort can be paraphrased thus: "the commodity prices are not the explanatory factor that provides the most appropriate cause of our economy's state". 29

${ }^{29}$ Someone might object that my Necessity-3 introduces the notion of conditional necessity, not the notion of absolute necessity. I do not believe that such an objection has a real point against my claim. There is some similarity between the logical structure underlying both usages of the expression "necessary". The similarity can be made explicit with a paraphrase such as this: "(i) if there is to be an appropriate explanation of this explanandum, then (ii) this is the principle necessary (for that explanation)". (1) However, an uncautious use of this paraphrase - as well as of what Aristotle says about conditional necessity in Metaphysics 1015b10, namely, that, in the case of things which are conditionally necessary, "the cause by which it is necessary is another thing"-will lead to a mistaken inversion in the grounding or explanatory order. It is true to say that our concern for attaining the appropriate explanation is what makes the necessary principle necessary for us or for our scientific knowledge. But even if nobody had ever realised the desirability to attain appropriate explanations, the real-world relation between the fact to be explained and its cause would still be there-independently of us-and the grounding connection between them would still be one from explanans (premises) to explanandum (conclusion), but not the other way around. Now, this means that the grounding relation goes from (ii) to (i): what is captured as a necessary- 3 principle is what makes our explanation turn out to be the most appropriate one. Now, in the most conspicuous appeals to conditional necessity (in natural teleology and in ethical reasoning), the grounding order is rather from (i) to (ii): 
Further, passage at 74 b21-26 will be in harmony with its immediate context if understood along Necessity-3: Aristotle's point is that acceptable or even true sentences are not enough for being principles. Principles must also be primary and appropriate. And the notion of "what is [most] appropriate" (oikeion, 74b26) is precisely what I am trying to depict as a necessary-3 principle, whose main feature is to capture what exactly its explanandum is as such. Appealing here to Necessity-2 would turn this passage into a "curious aside" (Barnes 1993, 126), not in harmony with its context.

\subsection{Compatibility with "For the Most Part" Sentences}

Another advantage of the triadic notion of a necessary principle is that it is also compatible with a demonstrative sentence being true for the most part (87b21-25, 96a8-19). Now, most (if not all) natural sciences for Aristotle seem to operate with sentences of this sort. Hence, the main source of the alleged mismatch between Aristotle's theory of demonstrative knowledge and Aristotle's real practice in the field of natural sciences will simply vanish on my account. Each sentence belonging to a natural science might well be true only for the most part (such as "every sheep has four legs"). But this does not prevent the middle term from being the necessary principle in the triadic sense: this middle term will be the required one for the fully appropriate explanation of why (say) sheeps have four legs (for the most part).

\section{Explaining $X$ Through X's Essence}

When Aristotle introduces his triadic model of explanation in APo, he claims that knowing the "what it is" is the same as knowing "why it is" (90a14-15; 93a4). If we grant that knowing why it is in this context is tantamount to hav-

the desire for an end is the ground for the desirability of the means; the structure of a house is the ground for the wall's being there etc. (2) Besides, the notion of "conditional necessity" introduced at Metaphysics 1015a20-26 seems to accommodate only conditions which are necessary but not sufficient for a desired result ("sunaitia"), whereas my notion of "necessary-3 principle" refers to items which are necessary and sufficient for the fully appropriate explanation. (3) Furthermore, this similarity highlighted above is not finegrained enough to capture one important aspect of my claim, which is the shift from a dyadic to a triadic-explanatory structure. Conditional necessity might still be used as a different way of understanding how a given sentence turns out to be necessary in the sense of a necessarily true proposition, but what most concerns me is that a "necessary principle" is called necessary not because of its being a necessarily true proposition, but because of its explanatory power in relation to an explanandum cast in a syllogistic framework. Thus, there are many more usages of "necessity/necessary" terminology in Aristotle's Greek than what is covered by the rough bipolarity between absolute and conditional necessity (see, e.g., Topics 155b19, 20, 29, 36; 156a10; 157a12; 159a2o; and Prior Analytics 42a39; 47a19; 53a35; 66a36: Aristotle introduces the notion of premises required for an intended conclusion). 
ing scientific knowledge, and that knowing what $X$ is amounts to knowing $X$ 's essence, Aristotle's claim implies that scientific knowledge of $X$ ("why $X$ is") is the same as the knowledge of $X$ 's essence ("what $X$ is"): this implied claim can be encapsulated in the lemma "explaining $X$ through $X$ 's essence".

Now, there are two ways of understanding the lemma "explaining $X$ through $X$ 's essence" in the threefold model of explanation. An explanandum is introducible by a nominal expression which packs the predicative relation between $C$ and $A .{ }^{30}$ Let $X$ be that nominal expression. Thus, to use one of Aristotle's favourite examples, the phenomenon which results from noise of a certain type $(A)$ being attributed to clouds $(C)$ is presented under the name "thunder" $(=X) \cdot{ }^{31}$ With " $X$ " standing for the explanandum as such, explaining $X$ through $X$ 's essence will follow what I call Version A of the lemma, which has the following distinctive features:

(a) The middle term $B$ is introduced either as the essence of the $X$-term, or as the essence of the $A$-term, but not as the essence of the $C$-term.

(b) The $A$-term, even if it is a necessary feature of $C$, is not appropriately explained by $C$ 's essence.

Version A is implied in all those passages and examples I have discussed in section 3 , and commitment to the notion of a necessary-3 principle, which is explanandum-sensitive, is tantamount to commitment to Version A. Before discussing Version A in more detail, let me present Version B, which can be characterized by the following distinctive features: $:^{32}$

$\left(a^{*}\right)$ The middle term $B$ is introduced as the essence of the $C$-term.

$\left(\mathrm{b}^{\star}\right)$ The $A$-term is introduced as a necessary feature of $C$, to be appropriately explained by $C$ 's essence.

In Version B, the lemma "explaining $X$ through $X$ 's essence" is understood in such a way that the role of " $X$ " is played by the $C$-term, not by the pragma $C A$ or by the attribute $A$. Version $\mathrm{B}$ is the one standardly acknowledged by contemporary discussions of essentialism. For instance, in

${ }^{30}$ See (Charles 2000, 64-67, 198-213), (Charles 2010, 289-292).

${ }^{31}$ At the end of the story, questions of the form "why does it thunder" must be rephrased not into "why does thunder occur in the clouds" (see 90a3, 93a3o, with the parallel case of "eclipse"), but rather into "why does noise (of a certain type) occur in the clouds" See Metaphysics 1041a23-26, APo 93b10-12. See (Williams and Charles 2013, 123), (Charles 2000, 283-294), (Lewis 2013, 276-277).

${ }^{32}$ See (Charles 2000, 245-265), (Williams and Charles 2013, 123-124). Version B is the one standardly acknowledged by contemporary discussions of essentialism, for instance (Fine 1994). I urge that Version A must be taken into account as well for attaining a more finegrained understanding of the explanatory role of essences. 
Fine's discussions, essences, as what determine the identity of their objects, are also the grounds in virtue of which a collection of other features belongs to those objects. ${ }^{33}$ Fine distinguishes between what belongs strictly to the essence of an object, its constitutive essence, and what follows from it-its consequential essence. Several issues might be addressed here and many details might be described with more exactitude, but for my purposes it is enough to highlight that Fine's picture seems to fit into Version $B$ better than into Version A: an object (the essence-bearer) plays the role of the $C$-term, the constitutive essence plays the role of the $B$-term and the consequential essence plays the role of the $A$-term. Now, both Versions can be claimed to be found in Aristotle's text, but preferences for Version B have almost led Version A to be overlooked. My concern is to defend Version A against this oversight. I have argued that what Aristotle says about necessary principles in APo commits him to Version A; I will now argue that Version A gives us an important standpoint for the essentialism debate: it stands against the idea that the Necessity-2 of a property is what makes that property essential to its subject and explanatory of its features.

Essentialism is often construed as a view in which the essentiality of a given property $P$ either flows from or is identical with its Necessity-2. The essence of an object is taken to be the set of properties that an object possesses in every possible world in which it exists. On this construal, the explanatoriness of the essence will also depend on its necessity; for it is only by pertaining to its object in every possible world that an essence would be able to explain anything about that object. ${ }^{34}$ Now, recent discussions have made a powerful case against such a construal. ${ }^{35}$ Philosophers have argued that it is rather the essentiality of a property $P$ that grounds not only its necessarily belonging to its subject, but also the Necessity-2 of other necessary features of the same subject. Thus, on this account, it is by being essential that an essential property belongs necessarily to its subject, ${ }^{36}$ but not the other way around; and it is by being essential that an essential property grounds and explains why its subject must have a necessary property.

Now, it might seem at first glance that my view runs against this picture, since my Version A acknowledges an explanatory property $B$ that, on the one hand, is not the essence of a given subject $C$, but, on the other hand, explains why $C$ has a given necessary property $A$. Thus, one might say that,

33 (Fine 1994, 3-5), (Fine 1995, 275-280).

${ }^{34}$ This is the standard picture of essentialism made popular by Quine. Lowe $(2008,34)$ has convincingly pointed out that this wrong construal of essentialism is shared by many of the contemporary anti-Quinean essentialists.

35 (Fine 1994), (Lowe 2008, 45), (Peramatzis 2011, 309-311), (Koslicki 2012).

${ }^{36}$ See discussion in (Williams and Charles 2013, 127-131). 
on Version $A$, it is not by being essential (to $C$ ) that property $B$ explains why its subject $C$ must have the necessary property $A$. However, this is a wrong impression. My account still supports the basic claim that essentiality is intrinsically interrelated with explanatoriness and grounds the Necessity-2 of necessary properties. First, it is by being essential (not to $C$, but to the explanandum as such) that property $B$ explains why its subject $C$ has property $A$; besides, a necessary-3 principle, even without being the essence of $C$, is still related to it (I will shortly explain how this is so).

Consider again one of my previous examples of a necessary-3 principle and its foil. In order to make reference easier, I will identify the $B$ - and $A$ terms with numerical indexes (I will also supply a description for what would be the essence of being mortal):

$$
\begin{aligned}
& C \text { = human; } \\
& B_{1}=\text { being rational animal; } \\
& B_{2}=\text { having vital functions depending ultimately on an unstable } \\
& \text { mixture of the four elements; } \\
& A_{1}=\text { being capable of becoming literate; } \\
& A_{2}=\text { being mortal }
\end{aligned}
$$

I have argued that $A_{2}$ is appropriately explained by $B_{2}$ and that $A_{1}$ is appropriately explained by $B_{1}$. Now, an attempt to explain $A_{1}$ by means of $B_{2}$ will be uncontroversially a failure (since the major premise of the syllogism will be false), while an attempt to explain $A_{2}$ through $B_{1}$ will deliver some explanation, but not the most appropriate one, which uniquely counts as the scientific explanation simpliciter. However, this last case will not affect the appropriateness of explaining $A_{1}$ by means of $B_{1}$. The fact that $B_{1}$ does not deliver the most appropriate explanation for $A_{2}$ does not affect the appropriateness of $B_{1}$ for the full explanation of $A_{1}$. I submit that this point can be generalised: for any given property such as $B_{1}$, which is essential to $C$, there are necessary properties such as $A_{1}$ which are appropriately explained by $B_{1}$ even if $B_{1}$ is not the principle necessary-3 for the fully appropriate explanation of other properties $A_{2}, A_{n}$ etc. A similar story holds for the other case as well: any given property such as $B_{2}$ is the principle necessary-3 for the fully appropriate explanation of a property such as $A_{2}$, but there are many necessary properties $A_{x}, A_{n}$ etc. of the same subject $C$ (e.g., capable of becoming literate) which are not appropriately explained by $B_{2}$. In both cases, it is by being essential that a property $B_{x}$ explains why its subject $C$ has some other property $A_{x}$. The difference is that $B_{1}$ is essential to its subject $C$, while $B_{2}$ is essential to the attribute $A_{2}$. Essences are still at the core of scientific 
explanations in both cases: the worry about my approach running against this point is not well founded.

It is important at this juncture to stress that Versions $\mathrm{A}$ and $\mathrm{B}$ are far from being incompatible or unconnected with each other. I will take up my previous examples to show this. A property such as $A_{2}$ is appropriately explained by $B_{2}$, which is not the essence of their subject, $C$. However, the explanatory property $B_{2}$ is not unrelated to $C$ 's essence. The important idea is that $B_{2}$ is connected with $B_{1}$ at some point. Having vital functions depending ultimately on an unsteady mixture of the four elements is something entailed by being a rational animal. I would say that the relation between $B_{1}$ and $B_{2}$ might be described as similar to Fine's relation between constitutive essence and restricted consequential essence. There are obvious differences, of course (for Fine's notion of restricted consequential essence has no reference to something like the essence of the attribute to be explained), but my purpose is just to highlight the entailment relation between the relata. In this way, inasmuch as $B_{1}$ is the essence of $C$ and $B_{1}$ entails $B_{2}, B_{1}$ furnishes some ground for $C$ 's having the property $A_{2}$. This, however, must not lead us to conclude that $B_{1}$ will be thereby the most appropriate explanatory factor for $A_{2}$. If I am correct, the notion of a necessary-3 principle involves uniqueness-for each explanandum $C A_{x}$, there is only one principle necessary for the most appropriate explanation - and this by itself shows that the relation of "being the necessary-3 principle of" is not transitive.

\section{Discussion of Some Objections}

One might object that my approach seems to inflate the population of essence-bearers. Aristotle's metaphysics seems to acknowledge only substances as primary essence-bearers, and even if the flexibility of his model for scientific explanation seems to make room for some attributes as (secondary) essence-bearers, it would be too much to say that any pragma articulated in a predicative tie, like human's being mortal, will be an essencebearer as well.

Against this worry, I start by answering that the introduction of these essence-bearers is grounded in Aristotle's text. The notion of essence I am talking about here is what Aristotle introduces with his expressions "what $X$ is" (to ti esti) and "what it is to be $X$ " (to ti èn einai). ${ }^{37}$. Now, Aristotle talks about what thunder is, and what he says at 93a12-13 implies that to ti $\bar{e} n$ einai should be ascribed to thunder. Consequently, thunder has an essence

\footnotetext{
${ }^{37}$ See $90 a 15$ ff., 90b30, 92a 34-35, $93 \mathrm{a} 4$ (for “to ti esti"); and 91a25-26, b8, 26, 92a13, 93a12-13, $94 \mathrm{a} 21$ (for "to ti en einai"). Sometimes Aristotle uses ousia to introduce the same notion (9ob3o-31; 91b9, 27; 92a34, b14; 96a34).
} 
at least in the sense in which essence corresponds to what is presented as an explanatory answer to the question "what is it?" and the notion of essence in APo seems to be nothing more than that. I do not mean that "essence" in APo stands for what is given in any sensible and enlightening answer to the question "what is it?". In order to display an essence, the explanatory answer must pick out the cause (in the Aristotelian sense) that makes the thing what it is and, therefore, fully accounts for its identity and its unity.

Another important qualification is due here: "thunder" is a name for the phenomenon articulated as the occurrence of certain type of noise in the clouds due to a certain regular and traceable cause. Thus, it is not that any pragma able to be articulated in a predication will be an essence-bearer, for it will be an essence-bearer only if the predicative tie at stake does have a regular cause. Now, if thunder-precisely as able to be articulated as the occurrence of certain feature in a given subject due to a certain regular cause-has an essence, why would any other phenomenon of the same type not have an essence as well?

The issue hinges on Aristotle's treatment of substance and essence in Metaphysics VII. Many scholars believe that Aristotle is arguing in Metaphysics VII that only substances are essence-bearers (or at least that only substances are the primary essence-bearers) and that accidental compounds such as pale man do not have essences. There is no room for a careful consideration of these issues within this paper, but it should be noted that those, who believe that Aristotle is restricting the population of essence-bearers in Metaphysics VII, must say something about Aristotle's treatment of essence in $A P o$, where Aristotle's talk of essence and explanation of $X$ by means of $X$ 's essence implies that regular events like thunder are acknowledged as essence-bearers. Aristotle does not show any interest in arguing that thunder's essence should be reduced to a substance's essence; nor does Aristotle argue that "thunder" has only a nominal definition. Quite on the contrary, he seems committed to the idea that thunder has a real essence which plays a major role in the scientific explanation of thunder. Now, much in this debate depends on whether essences are taken as entities themselves. ${ }^{38}$ Those who are worried about pragmata as essence-bearers seem to favour a positive answer. However, at least in APo, Aristotle's notion of essence can be taken to imply a negative answer: far from being new entities introduced in the

${ }^{38}$ For recent discussion about this topic, see (Lowe 2008, 34, 39), (Lowe 2013, 203), (Tahko 2013, 56-60). Code $(1985,110-113,119)$ ascribes to Aristotle the view that an object $X$ (of a proper kind) is the same as its own essence, which implies that essences are not introduced as new entities in the furniture of the world. Against this view, Charles (2011) has objected that identity with its bearer will prevent essences from having their explanatory and grounding role. 
furniture of the world, essences are just what their bearers exactly are. Definitions, as the linguistic counterparts of essences, do not introduce different entities, but just say how exactly and why exactly their bearers are what they are. ${ }^{39}$ Essences might be such that their full specification involves reference to other entities. Thus, thunder's essence involves reference to clouds and to fire; eclipse's essence involves reference to the Moon and to the Earth. But being fully specifiable only by reference to other entities does not imply that essences are entities themselves.

\section{Conclusion}

I have argued that Aristotle's notion of a necessary principle in his APo should not be understood in terms of a necessarily true sentence. A necessary principle should rather be understood in terms of explanatory appropriateness: a necessary principle is the principle that is strictly necessary for the fully appropriate explanation of a given explanandum. I have argued that this notion has philosophical advantages in its favour: it allows us a more fine-grained understanding of the exact factor on which the most appropriate explanation of a given explanandum depends. I have also argued that this notion of a necessary principle is in tune with recent developments in the essentialism debate as it promotes the idea that necessary properties are grounded in essential properties but not the other way around. Furthermore, from an exegetical standpoint, my account has the advantage of making sense of Aristotle's talk about "necessary [items]" in APo without charging him with false propositions like $B F P$ : "If $P$ is inferred from $\Pi$, and $\Pi$ is necessary, then $P$ is demonstrated". At a first glance, Aristotle seems to be committed to BFP in $\mathrm{T}_{1}$ (74b15-18). Such a commitment would be helpful to those who believe that not only Aristotle, but philosophers in general, are right in claiming that Necessity-2 is the basic ground to which the explanatoriness and the essentiality of a property should be reduced. However, I have shown that Necessity-3 cannot be reduced to Necessity-2 and cannot even be understood in terms of it. Aristotle does not say in $\mathrm{T}_{1}\left(74 \mathrm{~b}_{15}-18\right)$ that necessary predicative ties are by themselves the ground for scientific explanation and thereby (granting that scientific explanation appeals to essences) the ground for essences. Aristotle never endorsed BFP: "If $P$ is inferred from $\Pi$, and $\Pi$ is necessary, then $P$ is demonstrated". So much the better for Aristotle and

${ }^{39}$ Lowe $(2008,46)$ ascribes an essence to the event death of Socrates (as well as to other kinds of things) and I think he is right in doing that. I will not address the question whether Aristotle will go so far as to say with Tahko $(2013,56)$ that "non-existent entities can have essences" or "every metaphysically possible entity must have an essence". But I just want to point out that the acknowledgment of pragmata as essence-bearers in APo cannot be ignored and is a defensible and interesting philosophical view. 
much better for us.

\section{Acknowledgments}

Earlier drafts of this paper were read at the Workshop on Ancient Philosophy in the University of Oxford and in the University of Campinas (Unicamp). I am very grateful to audience members for their helpful questions and comments on those occasions, especially to David Charles, Michael Peramatzis, Thomas Johansen, Gail Fine, Terry Irwin, Stefan Sienkiewicz, Laura Castelli, Alan Code, Paolo Fait, David Bronstein, Marko Malink, Phil Corkum and Rodrigo Guerizoli. I owe a great debt to David Bronstein, Ryan Putzer, Frederique Janssen-Lauret, Riin Sirkel and an anonimous referee for many remarks and suggestions that improved the writing style of this paper.

\section{Bibliography}

Ackrill, J. L. (ed.) (1963). Aristotle's Categories and De interpretatione, Oxford University Press, Oxford.

Barnes, J. (1981). Proof and syllogism, in E. Berti (ed.), Aristotle on Science: The Posterior Analytics, Editrice Antenore, Padova, pp. 17-59.

Barnes, J. (ed.) (1993). Posterior Analytics, Oxford University Press, Oxford.

Burnyeat, M. F. (1981). Aristotle on understanding knowledge, in E. Berti (ed.), Aristotle on Science: The Posterior Analytics, Editrice Antenore, Padova, pp. 97-140.

Burnyeat, M. F. (2011). Episteme, in B. Morrison and K. Ieradiakonou (eds), Episteme, etc. Essays in Honour of Jonathan Barnes, Oxford University Press, Oxford, pp. 3-29.

Charles, D. (2000). Aristotle on Meaning and Essence, Oxford University Press, Oxford.

Charles, D. (2010). Definition and explanation in Posterior Analytics and Metaphysics, in D. Charles (ed.), Definition in Greek Philosophy, Oxford University Press, Oxford, pp. 286-328.

Charles, D. (2011). Some remarks on substance and essence in Aristotle's Metaphysics z.6, in B. Morrison and K. Ieradiakonou (eds), Episteme, etc. Essays in Honour of Jonathan Barnes, Oxford University Press, Oxford, pp. 150-171.

Code, A. (1985). On the origin of some Aristotelian theses about predication, in J. Bogen and J. McGuire (eds), How Things Are, Reidel, Dordrecht, 
pp. 101-131.

Corkum, P. (forthcoming). Is the syllogistic a logic, History and Philosophy of Logic .

Ferejohn, M. (1994). The immediate premises of Aristotelian demonstration, Ancient Philosophy 14: 79-97.

Fine, G. (2010). Aristotle's two worlds: Knowledge and belief in Posterior Analytics i.33, Proceedings of the Aristotelian Society CX: 323-346.

Fine, K. (1994). Essence and modality, Philosophical Perspectives 8: 1-16.

Fine, K. (1995). Ontological dependence, Proceedings of the Aristotelian Society 95: 269-290.

Goldin, O. (2013). Circular justification and explanation in Aristotle, Phronesis 58: 195-214.

Hankinson, R. J. (1998). Cause and Explanation in Ancient Greek Thought, Oxford University Press.

Koslicki, K. (2012). Essence, necessity and explanation, in T. Tahko (ed.), Contemporary Aristotelian Metaphysics, Cambridge University Press, Cambridge, pp. 187-206.

Kosman, L. A. (1973). Explanation, understanding and insight in Aristotle's Posterior Analytics, in Lee, Mourelatos and Rorty (eds), Exegesis and Argument, Assen, Van Gorcum.

Lesher, J. H. (2001). Aristotle on $\epsilon \pi \iota \sigma \tau \eta \mu \eta$ as understanding, Ancient Philosophy 21: 45-55.

Lewis, F. A. (2013). How Aristotle Gets by in Metaphysics Zeta, Oxford University Press, Oxford.

Lloyd, A. C. (1981). Necessity and essence in the Posterior Analytics, in E. Berti (ed.), Aristotle on Science: The Posterior Analytics, Editrice Antenore, Padova, pp. 157-171.

Lowe, E. J. (2008). Two notions of being: Entity and essence, Royal Institute of Philosophy Supplements 62: 23-48.

Lowe, E. J. (2013). Neo-aristotelian metaphysics: A brief exposition and defense, in E. Feser (ed.), Aristotle on Method and Metaphysics, Palgrave Macmillan, New York, pp. 196-205.

Malink, M. (2013). Aristotle's Modal Syllogistic, Harvard University Press, Cambridge.

Matthen, M. (1981). The structure of Aristotelian science, in M. Matthen (ed.), Aristotle Today: Essays on Aristotle's ideal of science, Academic Printing \& Publishing, Edmonton, pp. 1-23. 
McKirahan, R. (1992). Principles and Proofs: Aristotle's Theory of Demonstrative Science, Princeton University Press, Princeton.

Mignucci, M. (2007). Aristotele: Analitici Secondi, Ed. Laterza, Roma-Bari.

Peramatzis, M. (2010). Essence and per se predication in Aristotle's Metaphysics z4, Oxford Studies in Ancient Philosophy 39: 121-182.

Peramatzis, M. (2011). Priority in Aristotle's Metaphysics, Oxford University Press, Oxford.

Peramatzis, M. (n.d.). Aristotle on knowledge and belief: APo a-33. unpublished draft.

Ross, W. D. (ed.) (1949). Aristotle's Prior Analytics and Posterior Analytics, Oxford University Press, Oxford.

Smith, R. (1984). Immediate propositions and Aristotle's proof theory, Ancient Philosophy 6: 47-68.

Tahko, T. (2013). Metaphysics as first philosophy, in E. Feser (ed.), Aristotle on Method and Metaphysics, Palgrave Macmillan, New York, pp. 49-67.

Taylor, C. C. W. (1990). Aristotle's epistemology, in S. Everson (ed.), Epistemology, Cambridge University Press, Cambridge, pp. 116-142.

Williams, S. and Charles, D. (2013). Essence, modality and the master craftsman, in E. Feser (ed.), Aristotle on Method and Metaphysics, Palgrave Macmillan, New York, pp. 121-145. 